\title{
Equivalence of coupled networks and networks with multimodal frequency distributions: Conditions for the bimodal and trimodal case
}

\author{
Bastian Pietras, ${ }^{1,2, *}$ Nicolás Deschle, ${ }^{1,3}$ and Andreas Daffertshofer ${ }^{1, \dagger}$ \\ ${ }^{1}$ MOVE Research Institute Amsterdam \& Institute Brain and Behavior Amsterdam, Faculty of Behavioural and Movement Sciences, \\ Vrije Universiteit Amsterdam, van der Boechorststraat 9, Amsterdam 1081 BT, The Netherlands \\ ${ }^{2}$ Department of Physics, Lancaster University, Lancaster LA1 4YB, United Kingdom \\ ${ }^{3}$ Institute for Complex Systems and Mathematical Biology, University of Aberdeen, Kings College, Old Aberdeen AB24 3UE, United Kingdom
}

(Received 3 March 2016; revised manuscript received 16 August 2016; published 9 November 2016)

\begin{abstract}
Populations of oscillators can display a variety of synchronization patterns depending on the oscillators' intrinsic coupling and the coupling between them. We consider two coupled symmetric (sub)populations with unimodal frequency distributions. If internal and external coupling strengths are identical, a change of variables transforms the system into a single population of oscillators whose natural frequencies are bimodally distributed. Otherwise an additional bifurcation parameter $\kappa$ enters the dynamics. By using the Ott-Antonsen ansatz, we rigorously prove that $\kappa$ does not lead to new bifurcations, but that a symmetric two-coupled-population network and a network with a symmetric bimodal frequency distribution are topologically equivalent. Seeking for generalizations, we further analyze a symmetric trimodal network vis-à-vis three coupled symmetric unimodal populations. Here, however, the equivalence with respect to stability, dynamics, and bifurcations of the two systems no longer holds.
\end{abstract}

DOI: 10.1103/PhysRevE.94.052211

\section{INTRODUCTION}

The Kuramoto model is seminal for describing synchronization patterns in networks of phase oscillators. It has been investigated to great detail in numerous studies using different approaches; see, e.g., Refs. [1,2] for review. The analytical treatment typically relies on the formation of a common variable, the so-called order parameter, and seeks to pinpoint its dynamics. The more recently suggested ansatz by Ott and Antonsen [3] proved particularly fruitful for analyzing this dynamics. It applies to the thermodynamic limit, i.e., to infinitely large populations, and it contains major simplifications including the parametrization of the phase distribution's Fourier transform. Abrams and coworkers [4] were the first to describe the dynamics of two coupled populations using the Ott-Antonsen ansatz, confirming earlier results based on perturbation techniques [5,6]; see also Laing's extension including heterogeneity and phase lags [7]. Similarly, Kawamura and coworkers [8] derived a collective phase sensitivity function to describe synchronization across subpopulations, but they assumed only very weak coupling between them. A detailed bifurcation analysis of these dynamics without such restrictions, however, is still missing.

We discuss a network of two populations of Kuramoto oscillators with unimodally distributed natural frequencies. The dynamics will be compared with that of a single population of oscillators with bimodally distributed frequencies. The latter case has been extensively studied by Martens and coworkers [9]. Here we prove that a symmetric two-population network does fully resemble the case of one network with bimodally distributed frequencies. Assuming that the internal coupling strength (identical for both networks) can be distinct from the bidirectional external coupling strength, we introduce

\footnotetext{
*b.pietras@vu.nl

†a.daffertshofer@vu.nl
}

another degree of freedom in the dynamics, and by that go beyond a simple change of variables, which may transform the bimodal description into two populations. As we will show, this additional parameter does not lead to qualitatively different dynamics. Instead we prove the topological equivalence of the two systems.

A natural question is whether this equivalence can be generalized. For this we couple more than two populations and compare their dynamics to a network with a multimodal frequency distribution. We show that for a symmetric trimodal network vis-à-vis three subpopulations with identical internal coupling and identical (though distinct to the internal coupling strength) bidirectional external coupling, the dynamics already differ qualitatively from each other. Therefore, in the symmetric case considered here, the topological equivalence between coupled networks and networks with multimodal frequency distributions appears limited to two coupled networks vs one bimodal network, and fails when considering more than two subpopulations.

\section{REVISITING EXISTING THEORY}

The Kuramoto model displays the long-term dynamics of a system of $N \in \mathbb{N}$ weakly coupled limit-cycle oscillators, where each oscillator $k$ is fully described by its phase $\theta_{k}$. The latter evolves in time by following the dynamics

$$
\dot{\theta}_{k}=\omega_{k}+\frac{K}{N} \sum_{j=1}^{N} \sin \left(\theta_{j}-\theta_{k}\right) .
$$

Here, the natural frequencies $\omega_{k}$ are drawn from a distribution function $g(\omega)$, and $K$ denotes the strength of the all-toall-coupling between the oscillators. In his original work [10], Kuramoto assumed $g$ to be symmetric and centered around the origin thanks to the rotational invariance of the model. Introducing the notion of a complex-valued order 
parameter

$$
z=\frac{1}{N} \sum_{j=1}^{N} e^{i \theta_{j}}
$$

allows for measuring the degree of synchronization in the system. For the thermodynamic limit of infinitely many oscillators, $N \rightarrow \infty$, Kuramoto derived a critical coupling strength $K_{c}$ at which the incoherent solution, i.e., $z=0$, becomes unstable and a partially synchronized state, $z=$ const $\in(0,1]$, emerges [10]; see also Ref. [11]. In the case of a unimodal Lorentzian frequency distribution of width $\Delta$ and centered at $\omega_{0}=0$, this critical coupling is given by

$$
K_{c}=\frac{2}{\pi g(0)}=2 \Delta .
$$

In particular the onset, and in the following also the extent, of synchronized behavior depends crucially on both coupling strength $K$ and distribution width $\Delta$.

Of particular interest for our work is the coupling of two such Kuramoto networks. There we define two order parameters including a global one covering the entire network. Equivalently, we can decompose the global order parameter into local ones, each describing the dynamics of a single subpopulation. The interplay of these local order parameters has already been investigated some time ago. In 1991 Okuda and Kuramoto investigated the mutual entrainment of two oscillatory populations under the influence of noise [12]. All oscillators were assumed to have identical natural frequencies in their respective population and the resulting dynamics differed depending on coupling strength $K$, noise strength $D$, and the distance between the population-specific frequencies $\Delta \omega_{0}$. Next to a global incoherent and partially synchronized solution, they did not only find the existence of a steady state, which was later referred to as standing wave solution by Crawford [13], but numerical results revealed regimes of multistability, i.e., the coexistence of (at least) two stable solutions. Montbrió and coworkers [5] extended and generalized these findings by changing the setting slightly: Instead of letting the system be driven by noise, they assumed inhomogeneous natural frequencies drawn from unimodal distributions (per population). In the case of Lorentzians, they derived stability boundaries and illustrated their results for two coupled populations with numerical performance, and were among the first to discover chimera states, a notion that later that year had been introduced by Abrams and Strogatz [14] to denote regions of synchronization in an unsynchronized surrounding.

We would like to briefly comment on these two seemingly identical approaches: the first, in which the phase dynamics of identical oscillators is subject to noise, and the second, in which one considers heterogeneous oscillators without noise. As to the former, Okuda and Kuramoto assumed that the oscillators in each population have identical natural frequencies, i.e., $\omega_{\sigma, k}=\omega_{\sigma}$ for all $k=1, \ldots, N_{\sigma}$, and, in general, $\omega_{1} \neq \omega_{2}$. Let us rearrange their governing equation as follows [cf. Eq. (2.1) in Ref. [12] with $\Gamma(\phi)=\sin (\phi)$ and

$$
\begin{gathered}
\left.K^{(1)}=K^{(2)}=K\right]: \\
\dot{\theta}_{\sigma, k}=\omega_{\sigma}+\frac{K}{N} \sum_{\sigma^{\prime}=1}^{2} \sum_{j=1}^{N} \sin \left(\theta_{\sigma^{\prime}, j}-\theta_{\sigma, k}\right)+\xi_{\sigma, k}(t) \\
=\tilde{\omega}_{\sigma, k}+\frac{K}{N} \sum_{\sigma^{\prime}=1}^{2} \sum_{j=1}^{N} \sin \left(\theta_{\sigma, j}-\theta_{\sigma, k}\right),
\end{gathered}
$$

where $\tilde{\omega}_{\sigma, k}(t):=\left[\omega_{\sigma}+\xi_{\sigma, k}(t)\right]$, and $\xi_{\sigma, k}(t)=\xi_{k}(t)$ denote independent Gaussian noise processes with statistics $\left\langle\xi_{k}(t)\right\rangle=$ 0 and $\left\langle\xi_{k}(t) \xi_{j}\left(t^{\prime}\right)\right\rangle=2 D \delta_{k, j} \delta\left(t-t^{\prime}\right)$. As Sakaguchi argued in Ref. [15], in the thermodynamic limit the dynamics of the Langevin equations (4a) can be described by a FokkerPlanck equation, whose diffusion coefficient coincides with the noise strength $D$. Given that the $\xi_{\sigma, k}(t)$ are Gaussian noise terms, one can consider the population dynamics (4a) as an Ornstein-Uhlenbeck (OU) process. Then, the results by Okuda and Kuramoto [12] appear in a different light. OU processes possess a Lorentzian-shaped power spectrum. That is, assuming complex-valued relaxation rates $H_{\sigma}=-\Delta-i \omega_{\sigma}$, the power spectra of the corresponding $\mathrm{OU}$ processes read

$$
S_{\sigma}(\omega)=\frac{\Delta}{\left(\omega-\omega_{\sigma}\right)^{2}+\Delta^{2}},
$$

see Eq. (1.8.38) in Ref. [16]. In due course, this noise-driven approach dwelling on the Fokker-Planck equation is equivalent to the case of coupled phase oscillators with natural frequencies drawn from Lorentzian distributions. This equivalence becomes evident in the continuum limit of oscillators, a necessary assumption for deriving mean-field dynamics by both a Fokker-Planck formalism, see, e.g., Refs. [10,12,17], and by the Ott-Antonsen ansatz $[3,9,18]$. Hence, the following section can be understood as an analytic confirmation (and extension) of the numerical results by Okuda and Kuramoto [12], who assumed two symmetric $\delta$ peaks as their bimodal frequency distribution and allowed Gaussian noise processes to drive the system as in (4a). Note that this equivalence mentioned is only valid for the linearized dynamics. Indeed, this linearization is sufficient for characterizing fixed points and bifurcation boundaries. When, however, considering the fully nonlinear system with noise, the Ott-Antonsen ansatz, which the following analysis will heavily dwell on, no longer exhibits the exact dynamics, see, e.g., [19].

Before 2008, the general idea to analytically reveal the dynamical behavior of these systems was to investigate small perturbations of (the distribution function) of the incoherent state. Major simplifications for characterizing oscillatory systems arose with Ott and Antonsen's breaking idea to simplify the Fourier series of the oscillators' distribution functions [3]; see Sec. III. Their proof that the manifold of such a class of distribution functions does indeed capture the long-term dynamics of Kuramoto (and more general) models $[18,20]$ paved the way for the success of the Ott-Antonsen ansatz, see also Ref. [21]. Martens and coworkers [9] were the first to tackle a bimodal Kuramoto network with the new theory and revealed a thorough bifurcation diagram including stability properties of the corresponding solutions. Although the disguise of two coupled unimodal Kuramoto networks as 
a single network with natural frequencies following a bimodal distribution has often been claimed, above all in the Appendix of Ref. [9], a rigorous proof was not provided.

We consider two symmetric populations of $N$ phase oscillators $\theta_{\sigma, k}$ each, with $\sigma=1,2$ and $k=1, \ldots, N$. The oscillators have natural frequencies $\omega_{\sigma, k}$ distributed according to a Lorentzian $g_{\sigma}$ of width $\Lambda_{1}=\Lambda_{2}=\Lambda$ that are centered around $+\varpi_{0}$ and $-\varpi_{0}$, respectively. We assume all-to-all coupling within each population with strength $K_{\text {int }}$, and also all-to-all coupling across populations with strength $K_{\text {ext }}$. The corresponding dynamics obeys the form

$$
\begin{gathered}
\dot{\theta}_{\sigma, k}=\omega_{\sigma, k}+\frac{K_{\text {int }}}{N} \sum_{j=1}^{N} \sin \left(\theta_{\sigma, j}-\theta_{\sigma, k}\right) \\
+\frac{K_{\text {ext }}}{N} \sum_{j=1}^{N} \sin \left(\theta_{\sigma^{\prime}, j}-\theta_{\sigma, k}\right)
\end{gathered}
$$

with $\left(\sigma, \sigma^{\prime}\right)=(1,2)$ or $(2,1)$. Set $K_{\text {int }}=K_{\text {ext }}=K$, and let $\theta_{k}=\theta_{1, k}$ and $\theta_{N+k}=\theta_{2, k}$. Then, (5) reads

$$
\dot{\theta}_{k}=\omega_{k}+\frac{K}{2 N} \sum_{j=1}^{2 N} \sin \left(\theta_{j}-\theta_{k}\right), \quad k=1, \ldots, 2 N,
$$

with $\omega_{k}$ drawn from a bimodal distribution $g=\left(g_{1}+g_{2}\right) / 2$ with $g_{1,2}$ as defined earlier. This change of variables unveils the equivalence of both descriptions. Here, a crucial point is the assumption that the intrinsic coupling strength equals the external one. In the next section we will prove that both systems are topologically equivalent even if $\kappa=K_{\text {ext }} / K_{\text {int }} \neq 1$.

\section{TWO-POPULATION DYNAMICS WITH THE OTT-ANTONSEN ANSATZ}

To avoid confusion with the bimodal approach of Martens et al., we discriminate between internal and external coupling strengths $K_{\text {int }} \neq K_{\text {ext }}$. We consider the limit $N \rightarrow \infty$ and introduce continuous, time-dependent distribution functions $f_{\sigma}$ of the subpopulations' oscillators. The integral of $f_{\sigma}$ over phase and frequency defines the (local) order parameters

$$
z_{\sigma}=\int_{\mathbb{R}} \int_{0}^{2 \pi} f_{\sigma}(\omega, \theta, t) e^{i \theta} d \theta d \omega,
$$

i.e., a (circular) mean value for each population $\sigma$. The OttAntonsen ansatz [3] incorporates the $2 \pi$ periodicity of $f_{\sigma}$ and further simplifies its Fourier series to a single Fourier component $\alpha_{\sigma}(\omega, t)$, i.e.,

$$
f_{\sigma}(\omega, \theta, t)=\frac{g_{\sigma}(\omega)}{2 \pi}\left\{1+\left[\sum_{n=1}^{\infty} \alpha_{\sigma}(\omega, t)^{n} e^{i n \theta}+\text { c.c. }\right]\right\} .
$$

With the normalization

$$
\int_{0}^{2 \pi} f_{\sigma}(\omega, \theta, t) d \theta=g_{\sigma}(\omega):=\frac{\Lambda}{\pi} \frac{1}{\left(\omega-\omega_{\sigma}\right)^{2}+\Lambda^{2}},
$$

where $\omega_{1 / 2}= \pm \varpi_{0}$, the dynamics of the order parameters $z_{\sigma}$ reduces to

$\dot{z}_{\sigma}=-\left(\Lambda \mp i \varpi_{0}\right) z_{\sigma}+\frac{K_{\mathrm{int}}}{2} z_{\sigma}\left(1-\left|z_{\sigma}\right|^{2}\right)+\frac{K_{\mathrm{ext}}}{2}\left(z_{\sigma^{\prime}}-z_{\sigma}^{2} z_{\sigma^{\prime}}^{*}\right)$.
Since $g_{\sigma}(\omega)$ are continuous, nonconstant frequency distributions, the Ott-Antonsen manifold comprises the entire dynamics [18]. Next, we rewrite the order parameters as $z_{\sigma}=\rho_{\sigma} e^{i \phi_{\sigma}}$ such that with the assumed symmetry $\rho:=\rho_{1}=$ $\rho_{2}$ the system (7) transforms into

$$
\begin{aligned}
\dot{\rho} & =-\Lambda \rho+\frac{\rho}{2}\left(1-\rho^{2}\right)\left[K_{\text {int }}+K_{\text {ext }} \cos \psi\right] \\
\dot{\psi} & =2 \varpi_{0}-K_{\text {ext }}\left(1+\rho^{2}\right) \sin \psi ;
\end{aligned}
$$

here we introduced the mean relative phase between the subpopulations as $\psi=\phi_{2}-\phi_{1}$. Finally, we rescale the parameters by means of $\tau=K_{\text {int }} \cdot t, \kappa=K_{\text {ext }} / K_{\text {int }}, \Delta=2 \Lambda / K_{\text {int }}$ and $\omega_{0}=2 \varpi_{0} / K_{\text {int }}$, substitute $q=\rho^{2}$, and transform $q(t) \rightarrow q(\tau)$ as well as $\psi(t) \rightarrow \psi(\tau)$ if not stated otherwise [22]. Then, we find for $0<\rho \leqslant 1$

$$
\begin{aligned}
\dot{q} & =q[1-\Delta-q+\kappa(1-q) \cos \psi] \\
\dot{\psi} & =\omega_{0}-\kappa(1+q) \sin \psi ;
\end{aligned}
$$

from here on the dot notation refers to the derivative with respect to $\tau$. The system (9) resembles Eqs. (25) and (26) in Ref. [9] with the additional parameter $\kappa$. For $\kappa=1$ both systems agree entirely [23]. As we will show, the additional parameter $\kappa$ does not alter the qualitative bifurcation scheme of our network. Hence, we can understand the bimodal formulation as an equivalent representation of the network consisting of two symmetric subpopulations.

\section{A. Incoherent state}

Before discussing (9) in more detail, we briefly analyze the stability of the fully incoherent state $q=0$. Following Martens et al. [9], we linearize (7) around $z_{1}=z_{2}=0$ and find two pairs of degenerated eigenvalues

$$
\lambda_{1 / 3}=\lambda_{2 / 4}=1-\Delta \mp \sqrt{\kappa^{2}-\omega_{0}^{2}}
$$

expressed in the aforementioned, rescaled parameters. Given the rotational invariance of the incoherent state, we expected this degeneracy. The incoherent state is linearly stable if and only if the real parts of these eigenvalues are less than or equal to zero. Using $\kappa \geqslant 0$ and $\omega_{0} \geqslant 0$ we find the stability boundary as

$$
\Delta=1+\left\{\begin{array}{ll}
\sqrt{\kappa^{2}-\omega_{0}^{2}} & \text { for } \kappa \geqslant \omega_{0} \\
0 & \text { otherwise }
\end{array},\right.
$$

which can be confirmed by perturbing the uniform distribution $f(\omega, \theta, t)=(2 \pi)^{-1}$; see Montbrió and coworkers [5] or Okuda and Kuramoto [12]. Crossing this boundary for $\kappa \geqslant \omega_{0}$ corresponds to a degenerated transcritical bifurcation, while crossing the half line $\Delta=1$ resembles a degenerated supercritical Hopf bifurcation; see Fig. 1, where the red plane displays the Hopf bifurcation and the orange cone the transcritical one.

\section{B. Bifurcation analysis of the coherent state}

Coming back to the system (9) we realize that its fixed points satisfy $1-\Delta-q=\kappa(1-q) \cos \psi$ and $\omega_{0}=\kappa(1+$ $q) \sin \psi$. Combining these using $\cos ^{2} \psi+\sin ^{2} \psi=1$ yields 


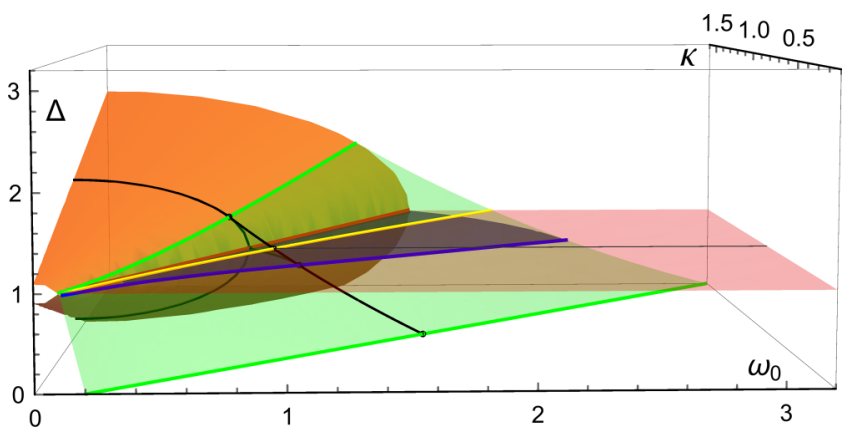

FIG. 1. Bifurcation boundaries. Red plane: Hopf; orange cone: transcritical; green plane (within green lines): saddle node; blue: homoclinic bifurcation. Blue line: Saddle-node loop curve; yellow: intersection of Hopf and $\mathrm{SN}$; black lines: cross section at $\kappa=0.8$, see also Fig. 3 .

$\kappa^{2}=[(1-\Delta-q) /(1-q)]^{2}+\left[\omega_{0} /(1+q)\right]^{2}$, or, equivalently,

$$
\omega_{0}= \pm \frac{1+q}{1-q} \sqrt{\Delta(2-2 q-\Delta)-\left(1-\kappa^{2}\right)(1-q)^{2}}
$$

as the implicit form of a hyperplane of fixed points $q_{s}=$ $q_{s}\left(\omega_{0}, \Delta, \kappa\right)$. After inserting $\partial \omega_{0} / \partial q=0$ in (12), the solution $\omega_{0}=\omega_{0}(\Delta, \kappa)$ forms a surface (green in Fig. 1) across which a saddle-node bifurcation appears. If both subpopulations contain oscillators with identical natural frequencies $\omega_{\sigma}$, i.e., if $\Delta=0$, then the saddle-node curve emerges from $\kappa=\omega_{0} / 2$. We stress this because in the literature the saddle-node curve has only been approximated numerically, while here we find that the Ott-Antonsen ansatz allows for deriving an analytical solution in a straightforward manner. The saddle-node plane starts at $\left(\omega_{0}, \Delta\right)=(2 \kappa, 0)$ and approaches tangentially the transcritical bifurcation plane at $\left(\omega_{0}, \Delta\right)=1 / 4\left(\sqrt{8 \kappa^{2}-2+2 \sqrt{1+8 \kappa^{2}}}, 3+\sqrt{1+8 \kappa^{2}}\right)$. This solution is consistent with the intersection point $\left(\omega_{0}, \Delta\right)_{\kappa=1}=$ $(\sqrt{3} / 2,3 / 2)$ reported in Ref. [9].

Can a change in $\kappa$ lead to new bifurcation behavior? To show that this is not the case, let $G_{1}\left(q, \psi ; \Delta, \omega_{0}, \kappa\right)$ denote the right-hand side of (9) and define $G_{2}\left(q, \psi ; \Delta, \omega_{0}, \kappa\right)=$ $\operatorname{det}\left\{\partial_{(q, \psi)} G_{1}\left(q, \psi ; \Delta, \omega_{0}, \kappa\right)\right\}$. For $\kappa=1$ it follows that

$$
\boldsymbol{G}\left(q, \psi ; \Delta, \omega_{0}, \kappa\right):=\left(\begin{array}{l}
G_{1}\left(q, \psi ; \Delta, \omega_{0}, \kappa\right) \\
G_{2}\left(q, \psi ; \Delta, \omega_{0}, \kappa\right)
\end{array}\right)=0
$$

along the saddle-node curve; cf. Eq. (33) in Ref. [9]. According to the implicit function theorem, there is no qualitative change in the $\left(\Delta, \omega_{0}\right)$-bifurcation diagram if

$$
\partial_{\kappa} \boldsymbol{G}\left(q, \psi ; \Delta, \omega_{0}, \kappa\right) \neq 0
$$

for any neutrally stable fixed point $\left(q, \psi ; \Delta, \omega_{0}, \kappa\right)=: \boldsymbol{x}$. Here, however, we have to extend this to a family of fixed points $\boldsymbol{x}_{s}=\boldsymbol{x}(\Delta)$ along the saddle-node curve parametrized by $\Delta$. Therefore, if (14) holds for a fixed point $\boldsymbol{x}_{1}$, i.e., if $\partial_{\kappa} \boldsymbol{G}\left(\boldsymbol{x}_{1}\right)$ $\neq 0$, then we still may end up at another point $\boldsymbol{x}_{2}$ on that curve. We circumvent this case by also requiring for any arbitrary $a \in \mathbb{R}$

$$
\partial_{\kappa} G_{1}\left(q, \psi ; \Delta, \omega_{0}, \kappa\right) \neq a \cdot \partial_{\Delta} G_{1}\left(q, \psi ; \Delta, \omega_{0}, \kappa\right)
$$

at every point along the saddle-node curve. Figure 2 shows that the inequality (14) holds for all $\boldsymbol{x}_{s}$. We note that, because
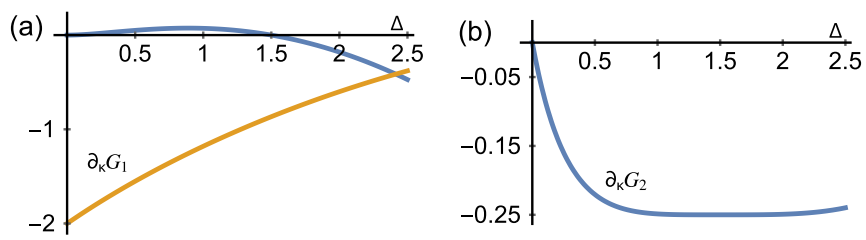

FIG. 2. Partial derivatives of $\partial_{K} G$ along the saddle-node plane at $\kappa=1$; (a) first and second component of $\partial_{\kappa} G_{1}(\Delta)$, (b) $\partial_{\kappa} G_{2}(\Delta)$.

$\dot{\psi}$ is independent of $\Delta$, it suffices to consider only the second equation of $\partial_{\kappa} G_{1}$, which is nonzero for $0 \leqslant \Delta<4$. That is, the bifurcation diagram is persistent against (small) perturbations around $\kappa=1$ and there are no bifurcations of codimension larger than 2 .

\section{MULTISTABILITY AND OSCILLATORY REGIMES}

For codimension 2, Martens and coworkers suggested the existence of saddle-node loop bifurcation points on the saddlenode plane below the Hopf bifurcation that can be identified numerically. In fact, the reduced dynamics (9) has a Jacobian along the saddle-node plane that is (conjugate to) a diagonal matrix with only one zero eigenvalue in the parameter range under study. This underlines the saddle-node character of that plane, but more importantly, it shows that these equations cannot be exploited for bifurcation points of codimension 2 .

In Appendix A we provide more details of the bifurcation scheme including numerical simulations. The latter demonstrate the existence of a multistability region; cf. Fig. 3 and Martens et al.'s Figs. 5 and 7(a). Multistability has been reported independently in Refs. $[5,7,9,12]$. The red parameter

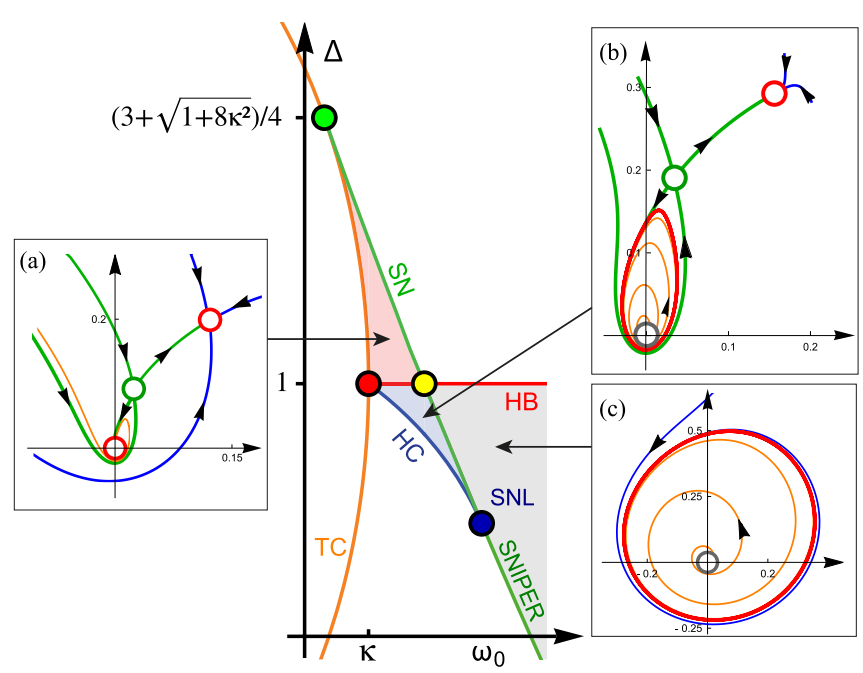

FIG. 3. Bifurcation boundaries: cross section of Fig. 1 at $\kappa<1$; red: Hopf; orange: transcritical; green: saddle node; blue: homoclinic; blue point: saddle-node loop bifurcation. Insets: $(q, \psi)$-phase portraits (in polar coordinates) in their specific parameter regions, red circle: stable fixed point; gray: unstable fixed point; green: saddle point. The bistability region (red/blue) overlaps with the oscillatory regime (blue/gray). (a) Coexistence of two stable fixed points, (b) a stable fixed point outside a stable limit cycle, (c) the more regular, stable limit cycle away from the SN curve. 
region, bounded by the transcritical cone (orange curve), the Hopf plane (red), and the saddle-node plane (green), reveals the coexistence of another stable, but nontrivial fixed point next to the stable incoherent solution (separated by a saddle point). In the blue parameter region left to the saddle-node plane and below the red Hopf plane, the incoherent solution has undergone a supercritical Hopf bifurcation such that a stable limit cycle coexists with the pair of stable fixed and saddle points. For the transverse stability properties of our solutions, i.e., stability against perturbations off the symmetry $\rho_{1}=\rho_{2}$, we refer to Sec. IV in Ref. [9]. Due to the equivalence of both the bimodal and the two subpopulation system, the stability results there can be readily adopted. Note that the equivalence also holds when introducing small time delays; see Appendix B.

Particularly interesting for future applications are the limit cycle oscillations in the $(q \cos \psi, q \sin \psi)$ plane shown in Figs. 3(b) and 3(c). There, both $q(t+T)=q(t)$ and $\psi(t+T)=\psi(t) \bmod 2 \pi$ hold for all $t \in \mathbb{R}$ given a fixed period length $T=T\left(\Delta, \omega_{0}, \kappa\right)$. We study these oscillations in more detail by introducing the global complex-valued order parameter $z=\left(z_{1}+z_{2}\right) / 2$, whose magnitude $|z|=R$ reads [24]

$$
R=\frac{\rho}{\sqrt{2}} \sqrt{1+\cos \psi}
$$

with $\rho=\sqrt{q}$. If $\dot{\psi}(t) \neq 0$, then $R(t)$ will oscillate. We would like to note that in this case oscillations in $R$ would be even observable without $q$ being periodic. However, for all parameter values outside the oscillatory regime, the dynamics contains stable fixed points at which obviously $\dot{\psi}=0$, i.e., $R \rightarrow$ const. As can be seen in Fig. 3(b), the limit cycle is deformed: it is neither circular nor symmetric about the origin. Then, also $q$ oscillates, i.e., not only the global order parameter $R$ oscillates, but so do the local ones $\rho=\rho_{1}=\rho_{2}$. For larger $\omega_{0}$ the limit cycle gains symmetry, but does not become a perfect circle. Hence oscillations contain higher harmonics; see Fig. 3(c). Future studies will address more details of the parameter dependency of frequency and amplitude of the $\rho$ and $R$ oscillations as well as their phase difference.

\section{MULTIMODAL VS MULTIPLE COUPLED NETWORKS}

Given that two coupled networks and networks with bimodal frequency distributions are equivalent, it appears obvious to search for generalizations. Can we derive a similar equivalence, as before, between multiple coupled unimodal networks and networks with symmetric multimodal frequency distributions? Anderson and coworkers studied communities of oscillators in systems with multiple subpopulations in Ref. [25]. They included mixes of attractive and repulsive couplings (in our notation $K_{\text {int }}$ and $K_{\text {ext }}$ should differ in sign) rendering the dynamics too diverse for analytical treatment. Closer to our approach, however, is the work by Komarov and Pikovsky [26] who showed a variety of synchronization characteristics as well as the emergence of chaotic states in the case of three positively coupled subpopulations. Thereby, they extended the numerical results for a trimodal network driven by noise [27]; see also our comment above about noise-driven networks with $\delta$ functions as frequency distributions.
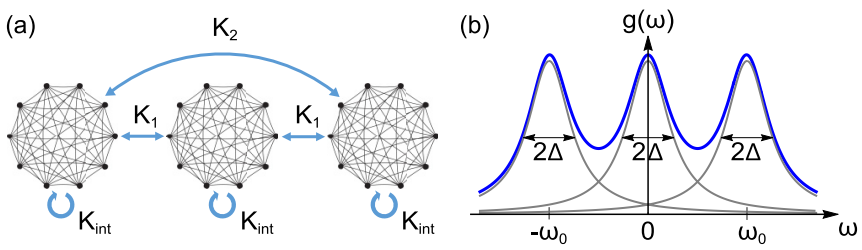

FIG. 4. (a) Three all-to-all coupled networks; (b) symmetric trimodal frequency distribution function.

We sketch the case of three subpopulations with a unimodal Lorentzian frequency distribution each: $g_{\sigma}(\omega)=$ $(\Lambda / \pi) /\left[\left(\omega-\left(-\varpi_{0}, 0,+\varpi_{0}\right)\right]^{2}+\Lambda^{2}\right)$ with peaks at $\left(-\varpi_{0}, 0,+\right.$ $\left.\varpi_{0}\right)$ [28]. This is compared with oscillators with a symmetric trimodal frequency distribution: $g(\omega)=\beta \cdot g_{1}(\omega)+\alpha \cdot g_{2}(\omega)+$ $\beta \cdot g_{3}(\omega)$ with $\alpha=\left(4 \varpi_{0}^{2}-2 \Lambda^{2}\right) /\left(12 \varpi_{0}^{2}\right)$, and $\beta=\left(4 \varpi_{0}^{2}+\right.$ $\left.\Lambda^{2}\right) /\left(12 \varpi_{0}^{2}\right)$ [29]. The two systems read

$$
\begin{aligned}
\dot{\theta}_{k} & =\omega_{k}+\frac{K}{3 N} \sum_{j=1}^{3 N} \sin \left(\theta_{j}-\theta_{k}\right) \\
\dot{\theta}_{\sigma, k} & =\omega_{\sigma, k}+\sum_{\tau=1}^{3} \frac{K_{\sigma, \tau}}{N} \sum_{j=1}^{N} \sin \left(\theta_{\tau, j}-\theta_{\sigma, k}\right),
\end{aligned}
$$

where $K_{\sigma, \tau}=K_{|\sigma-\tau|}$ with $K_{0}$ denoting the internal coupling strength $K_{\text {int }}$ within each population, $K_{1}$ the coupling strength between adjacent populations, and $K_{2}$ that between distant populations, see Fig. 4. In (17a) we have $k=1, \ldots, 3 N$, while in (17b) $k=1, \ldots, N$ and $\sigma=1-3$. When considering the thermodynamic limit, however, both systems consist of a continuum of oscillators. As before, we introduce (local) order parameters $z_{\sigma}=\rho_{\sigma} e^{i \phi_{\sigma}}$. Since the two outer populations are considered symmetric, we use $\rho_{13} \equiv \rho_{1}=\rho_{3}$ and $\phi_{2}-\phi_{1}=$ $\phi_{2}-\phi_{3}:=\psi$. By this we find the dynamics of (17a) after rescaling $\tau=(K / 2) \cdot t$ and $\omega_{0}=2 \varpi_{0} / K$ and $\Delta=2 \Lambda / K$ and $\kappa_{\alpha}=\alpha$ and $\kappa_{\beta}=\beta$ as

$$
\begin{aligned}
\dot{\rho}_{13} & =\rho_{13}\left[-\Delta+\left(1-\rho_{13}^{2}\right)\left(\kappa_{\alpha} \frac{\rho_{2}}{\rho_{13}} \cos \psi+\kappa_{\beta}(1+\cos 2 \psi)\right)\right] \\
\dot{\rho}_{2} & =\rho_{2}\left[-\Delta+\left(1-\rho_{2}^{2}\right)\left(\kappa_{\alpha}+2 \kappa_{\beta} \frac{\rho_{13}}{\rho_{2}} \cos \psi\right)\right] \\
\dot{\psi} & =\omega_{0}-\left(1+\rho_{13}^{2}\right)\left(\kappa_{\alpha} \frac{\rho_{2}}{\rho_{13}} \sin \psi+\kappa_{\beta} \sin 2 \psi\right) .
\end{aligned}
$$

Accordingly, we rescale system (17b) using $K=K_{\text {int }}+K_{1}+$ $K_{2}$ and $\tau=(K / 2) \cdot t, \Delta=2 \Lambda / K, \omega_{0}=2 \varpi_{0} / K$ and abbreviate $\kappa_{\alpha, \beta}=2 K_{1,2} / K$, which yields

$$
\begin{aligned}
\dot{\rho}_{13} & =\rho_{13}\left[-\Delta+\left(1-\rho_{13}^{2}\right)\left(\kappa_{0}+\kappa_{\alpha} \frac{\rho_{2}}{\rho_{13}} \cos \psi+\kappa_{\beta} \cos 2 \psi\right)\right] \\
\dot{\rho}_{2} & =\rho_{2}\left[-\Delta+\left(1-\rho_{2}^{2}\right)\left(\kappa_{0}+2 \kappa_{\alpha} \frac{\rho_{13}}{\rho_{2}} \cos \psi\right)\right] \\
\dot{\psi} & =\omega_{0}-\left(1+\rho_{13}^{2}\right)\left[\kappa_{\alpha} \frac{\rho_{2}}{\rho_{13}} \sin \psi+\kappa_{\beta} \sin 2 \psi\right]
\end{aligned}
$$

where $\kappa_{0}=1-\kappa_{\alpha}-\kappa_{\beta}$. Both systems can display a richer dynamical behavior than the dynamics (9) since they, e.g., contain coupling terms of first and second harmonics, that 


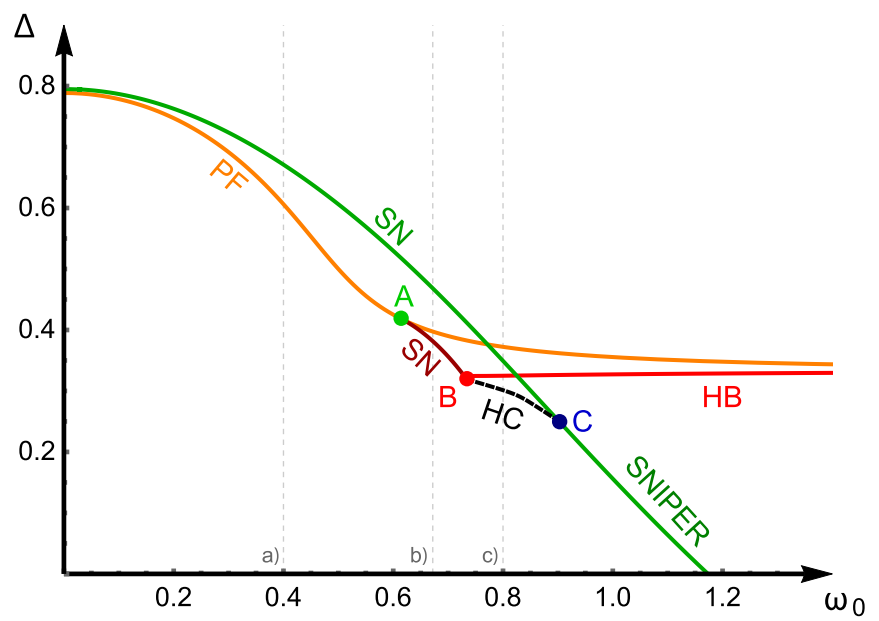

FIG. 5. Bifurcation boundaries of the symmetric trimodal network. Curves display a pitchfork (PF, orange); Hopf (HB, red); saddle-node (SN, green and dark red); SNIPER (green); and homoclinic (HC, black) bifurcation. Points denote codimension 2 bifurcations: Cusp (A), Bogdanov-Takens (B), and saddle-node loop (C).

may result in a $2: 1$ phase synchronization. When it comes to linking the two, we realize that they are only identical for the special case

$$
\kappa_{\alpha}=\kappa_{\beta}=\frac{1}{3} \Rightarrow \alpha=\beta .
$$

As $\alpha$ and $\beta$ only differ by $\Lambda^{2} /\left(4 \varpi_{0}\right)$, this implies $\Lambda \rightarrow 0$, hence the distribution function will consist of three $\delta$ peaks and the inhomogeneity is strongly reduced. As a consequence, the Ott-Antonsen manifold may not exhibit the whole dynamics of our system [18] and our description may remain incomplete, as has been found by Martens in Ref. [30] for even stronger symmetry assumptions in a network of three populations, though including phase lags. This is an arguably heuristic way of saying. In the following, we would therefore like to show that for our symmetric setup the dynamics of the two systems indeed differ qualitatively from each other.

Both systems can be described by the governing equations for $\rho_{13}, \rho_{2}$, and $\psi$. This enabled us to reduce the originally six-dimensional dynamics with $z_{j} \in \mathbb{C}$ to three dimensions. Furthermore, the control parameters are $\Delta$ and $\omega_{0}$, and the coupling parameters are $\kappa_{\alpha}$ and $\kappa_{\beta}$. In the symmetric trimodal case, the latter two are already fully described by the corresponding control parameters, i.e., $\kappa_{\alpha, \beta}=\kappa_{\alpha, \beta}\left(\Delta, \omega_{0}\right)$. Thus, the bifurcation diagram is two-dimensional. In contrast, in the three-network case we are free to choose $\kappa_{\alpha}, \kappa_{\beta}$ as long as they fulfill $0 \leqslant \kappa_{\alpha, \beta}<1$ and $0 \leqslant \kappa_{\alpha}+\kappa_{\beta}<1$. This implies that the bifurcation diagram becomes four-dimensional and we may be confronted with bifurcations of codimension higher than 2 .

\section{A. Symmetric trimodal network}

We first analyze the trimodal system with respect to fixed points and their stability, which leads us to the bifurcation diagram presented in Fig. 5. We consider $\left(\rho_{13}, \rho_{2}, \psi\right)$ as cylindrical coordinates with $\rho_{13,2} \in[0,1]$ and $\psi \in[0,2 \pi) ; \rho_{2}$ represents the height of the cylinder. For our symmetry assumptions, these variables fully represent the order parameter dynamics of the system (17a) away from the incoherent solution

$$
z=\frac{1}{3}\left(z_{1}+z_{2}+z_{3}\right) \equiv 0
$$

since for $z_{j}=0$ the phases $\phi_{j}$, and hence $\psi$ are not defined. Nevertheless, the cylindrical dynamics (18) still indicate the origin $\rho_{13}=0=\rho_{2}$ as a fixed point, so that the dynamical picture remains valid for $\rho_{13,2} \geqslant \epsilon>0$ with $\epsilon$ arbitrary small. The system exhibits the symmetry $\left(\rho_{13}, \rho_{2}, \psi\right) \mapsto\left(-\rho_{13},-\rho_{2}, \psi\right)$, such that the cylinder defined above can be point mirrored about the origin to $\rho_{2} \in[-1,0]$. In due course, bifurcation points as well as bifurcating branches off the incoherent solution will always appear in pairs $\left( \pm \rho_{13}^{*}, \pm \rho_{2}^{*}, \psi^{*}\right)$.

Having this said, we can focus on the bifurcation diagram Fig. 5. The orange curve denotes a pitchfork (PF) bifurcation of the incoherent solution $z=0$, at which it loses stability for $\Delta<\Delta_{P F}\left(\omega_{0}\right)$. Point $\mathrm{A}=\left(\omega_{A}, \Delta_{A}\right) \approx(0.614,0.418)$ (green) on the curve denotes the point where the PF bifurcation changes from subcritical $\left(\omega_{0}<\omega_{A}\right)$ to supercritical $\left(\omega_{0}>\omega_{A}\right)$. Let us first consider the parameter region where the PF bifurcation is subcritical, see, e.g., the dashed gray vertical line (a). At the PF point there are two unstable solution branches bifurcating off the incoherent solution $\left(\Delta>\Delta_{P F}\right)$, which gain stability via a saddle-node $(\mathrm{SN})$ bifurcation (green curve). Between the SN and the PF curves we find bistability of the stable incoherent solution together with a nontrivial fixed point - the branch with $\rho_{2}<0$ is not a physical solution as here the global order parameter has negative absolute value, $|z|<0$.

Beyond point $\mathrm{A}$ the incoherent solution undergoes a supercritical PF bifurcation $\left(\omega_{0}>\omega_{A}\right)$. The stable branches can then either lose stability via a SN bifurcation (dark red, $\omega_{0}<\omega_{B}$ ), which will be regained via a second SN bifurcation at the green curve, or the branches undergo a Hopf bifurcation (HB), see the red curve, beyond which we have oscillations of the order parameter. The point B (red), which distinguishes the two cases, is a Bogdanov-Takens point (codimension 2). Interestingly, oscillations can also cease. One possibility for this is that the unstable branch of the (green) SN bifurcation coalesces with the limit cycle, leading to a homoclinic (HC) bifurcation (black, dashed). The other possibility is that the $\mathrm{SN}$ bifurcation takes place directly on the limit cycle, leading to a saddle-node infinite period (SNIPER) or a saddle-node invariant circle (SNIC) bifurcation (green). The point $\mathrm{C}$ (dark blue), where the SN, HC, and SNIPER curves meet, is referred to as a saddle-node loop bifurcation; see also the discussion above for two coupled networks, Sec. IV.

Alternatively, we can characterize solutions via the behavior of the (global) order parameter $z(t)$, which lives in the complex unit disk. To compare our results with Ref. [27], we focus on the absolute value $R(t)=|z(t)| \in \mathbb{R}$ that reads in the cylindrical variables

$$
R(t)=\frac{1}{3} \sqrt{2 \rho_{13}^{2}+\rho_{2}^{2}+4 \rho_{13} \rho_{2} \cos \psi} .
$$

Figure 6 displays the typical behavior of $R$ along the dashed gray vertical lines [Figs. 5(a)-5(c)].

Since we are only interested in physical solutions, we concentrate on $R(t) \in[0,1]$. For small values of $\omega_{0}<\omega_{A} \approx 0.614$ [in Fig. 6(a) we used $\omega_{0}=0.4$ ] there is a 

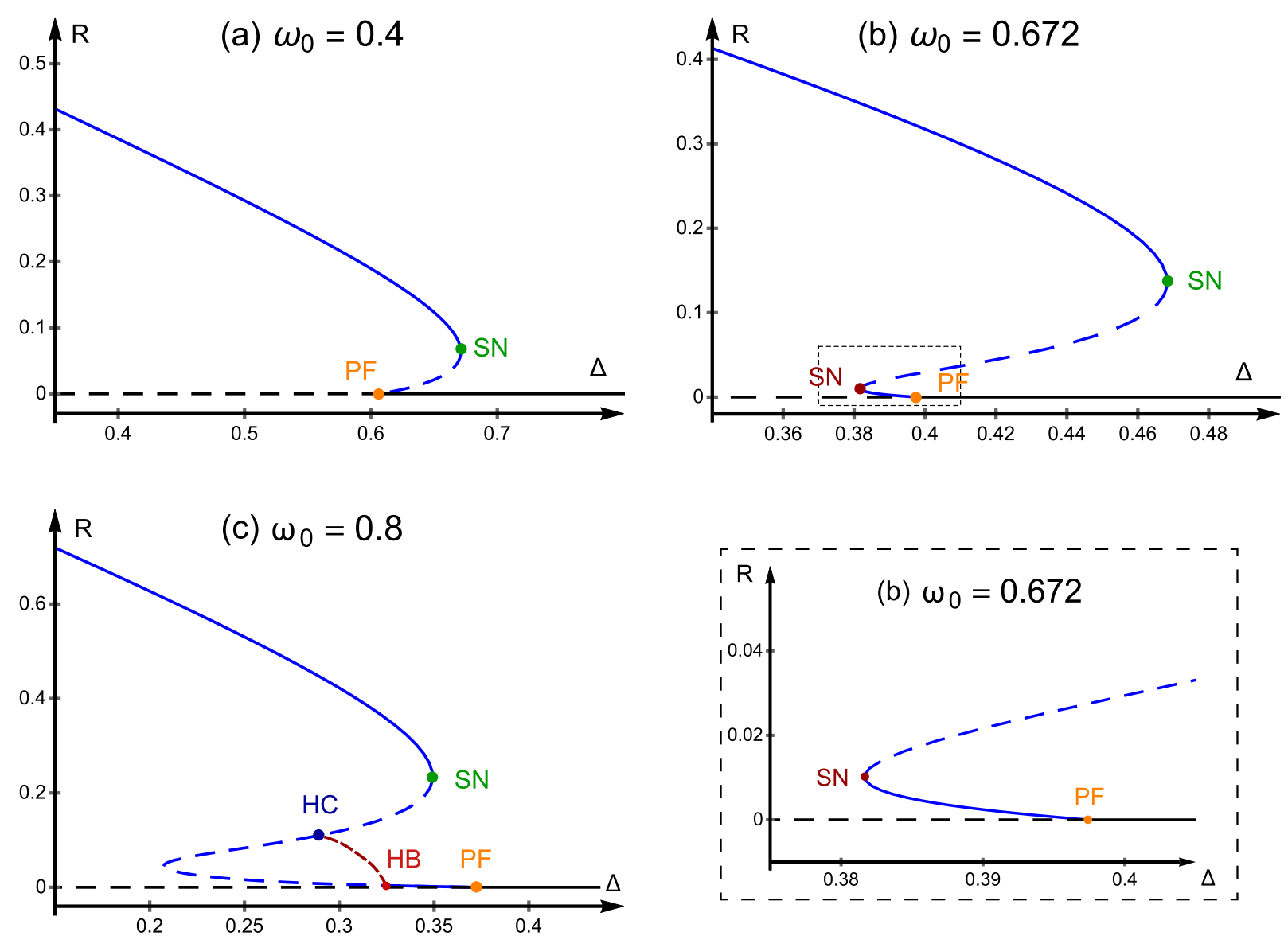

FIG. 6. Order parameter $R$ versus $\Delta$ for fixed $\omega_{0}$ according to the dashed lines [(a), (b), (c) in Fig. 5]. Solid lines denote stable, dashed lines unstable fixed points. (c) Dark red line denotes maximum amplitude of the (stable) limit cycle around the unstable fixed point. When the upper unstable fixed point coalesces with the limit cycle, oscillations cease in an homoclinic (HC) bifurcation.

subcritical pitchfork bifurcation (orange dot), where $R \equiv 0$ loses stability. The off-branching solution is first unstable and gains stability at the saddle-node point ( $\mathrm{SN}$, green). For $\Delta_{P F} \leqslant \Delta_{S N}$ we find multistability of two fixed points. In Fig. 6(b) we consider $\omega_{0}=0.672>\omega_{A}$. Here, the PF bifurcation of $R \equiv 0$ is supercritical. The nontrivial stable solution loses stability at the first SN point (dark red), before it regains stability at the second SN point (green). During this snaking behavior, we find multistability of the incoherent solution with a nontrivial solution for $\Delta_{P F} \leqslant \Delta \leqslant \Delta_{S N \text {, green, }}$ and of two nontrivial solutions for $\Delta_{S N \text {, red }} \leqslant \Delta \leqslant \Delta_{P F}$. This is typical near cusp bifurcations, because of which point $\mathrm{A}$ in Fig. 5 can be considered a (degenerate) cusp point. For even larger $\omega_{0}$, e.g., $\omega_{0}=0.8$ in Fig. $6(\mathrm{c})$, the incoherent solution loses stability at $\Delta_{P F}$ and then the stable branch undergoes a Hopf bifurcation (HB, red dot). In between, a SN bifurcation appeared at $\Delta_{S N}$, where the stable branch is monotonic increasing and the unstable branch decreases until it touches the limit cycle at $\Delta_{H C}$. At this point the oscillations, whose upper bound is depicted as a red dashed curve, cease in a homoclinic bifurcation.

We would like to remark that our findings confirm earlier results by Acebrón and coworkers [27]. Furthermore, we extend the theory for a symmetric trimodal Kuramoto model with a qualitative bifurcation analysis of all the fixed points. In particular, all bifurcation boundaries found in Fig. 5 could be derived analytically (except for a numerical approximation of the HC curve), which again manifests the capacity of the OA ansatz.

\section{B. Three coupled symmetric networks}

With a proper bifurcation diagram of the symmetric trimodal network at hand, we now focus on the network consisting of three all-to-all coupled symmetric populations each with a unimodal frequency distributions, see schematic in Fig. 4(a). The external coupling strengths $K_{1,2}$ for near and distant interactions across subpopulation boundaries, respectively, led to two additional bifurcation parameters $\kappa_{\alpha, \beta}$ in the order parameter dynamics. Using the symmetry assumptions as presented above, we are able to describe this dynamics as a three-dimensional system of coupled ODEs with in total four bifurcation parameters. A description of the full bifurcation scheme is beyond the scope of the paper. However, in order to disprove the claim that three coupled networks and the trimodal network are topologically equivalent, at least in the symmetric case considered here, it suffices to present a counterexample.

We consider again the cylindrical coordinates $\left(\rho_{13}, \rho_{2}, \psi\right)$, whose dynamics are given by (19). Transforming them into Euclidean coordinates $(x, y, z)$ in the cylinder

$$
Z=\left\{(x, y, z) \in \mathbb{R}^{3} \mid 0 \leqslant x^{2}+y^{2} \leqslant 1 \text { and } 0 \leqslant z \leqslant 1\right\}
$$




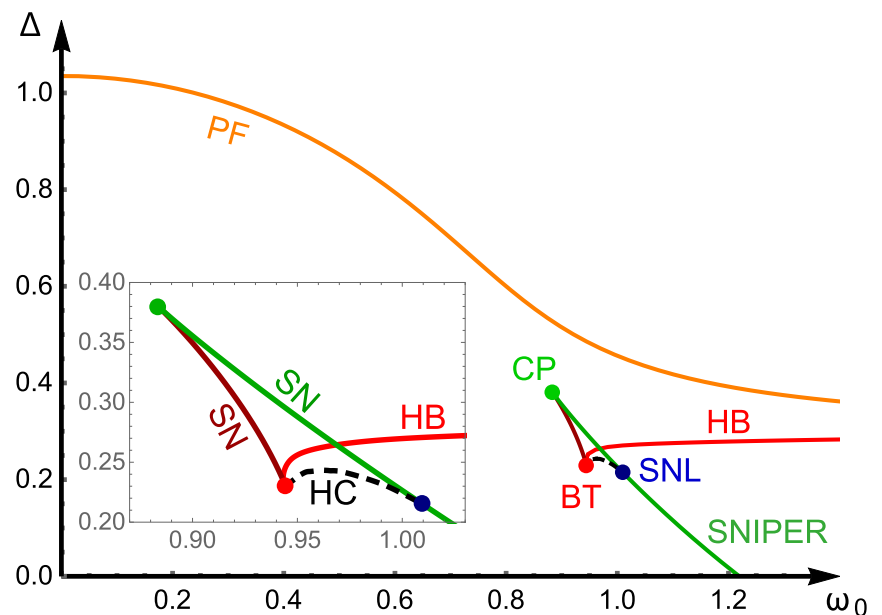

FIG. 7. Bifurcation boundaries of three coupled symmetric networks with coupling parameters $\kappa_{\alpha}=0.4$ and $\kappa_{\beta}=0.3$. Colors and abbreviations correspond to those in Fig. 5.

with $x=\rho_{13} \cos \psi, y=\rho_{13} \sin \psi$ and $z=\rho_{2}$, the dynamics in Euclidean space read

$$
\begin{aligned}
\dot{x}= & -\Delta x-\omega_{0} y+\left(1-\kappa_{\alpha}-2 \kappa_{\beta}\right)\left(1-x^{2}-y^{2}\right) \\
& +\left(1-x^{2}+y^{2}\right)\left(\kappa_{\alpha} z+2 \kappa_{\beta} x\right), \\
\dot{y}= & -\Delta y+\omega_{0} x+\left(1-\kappa_{\alpha}-2 \kappa_{\beta}\right)\left(1-x^{2}-y^{2}\right) \\
& -2 \kappa_{\alpha} x y z-4 \kappa_{\beta} x^{2} y \\
\dot{z}= & -\Delta z+\left(1-z^{2}\right)\left[\left(1-\kappa_{\alpha}-\kappa_{\beta}\right) z+2 \kappa_{\alpha} x\right] .
\end{aligned}
$$

For $\kappa_{\alpha}+2 \kappa_{\beta} \neq 1$ the origin $(0,0,0)$ is no longer a fixed point of the transformed system (22). This shows that the introduction of polar coordinates $z_{j}=\rho_{j} e^{i \phi_{j}}$ is only valid away from the incoherent solution $z_{j}=0=\rho_{j}$ for all $j=1,2,3$. Note that for the full six-dimensional dynamics, the incoherent solution $z=\left(z_{1}+z_{2}+z_{3}\right) / 3 \equiv 0$ is always a solution. However, the subsequent transformations into polar, cylindrical, and Euclidean coordinates show that the reflection symmetry as in the trimodal case breaks down in three-population approach when we choose coupling parameters off the line $\left\{\kappa_{\alpha}+2 \kappa_{\beta}=1\right\}$. Hence, we expect already here qualitative changes of the bifurcation boundaries from those obtained in the trimodal case.

Moreover, we can detect a qualitative difference for more similar settings, i.e., when reflection symmetry is maintained. Therefore, we assume in the following that $\kappa_{\alpha}+2 \kappa_{\beta}=1$. In fact, the $\kappa_{\alpha, \beta}$ of the trimodal network do fulfill this property. A bifurcation analysis of system (22) with respect to fixed points and their stability equivalent to Sec. V A reveals the following bifurcation diagram Fig. 7.

Note that here we fixed the coupling parameters to $\kappa_{\alpha}=0.4$ and $\kappa_{\beta}=0.3$. Nonetheless, we consider this example representative. We achieved similar bifurcation diagrams for a broad variety of parameter choices, even if we allowed $\kappa_{\alpha, \beta}$ to depend on $\Delta$ and $\omega_{0}$ as in the trimodal case. Comparing Figs. 5 and 7, one recognizes similar bifurcations, such as a pitchfork (PF, orange); a Hopf (HB, red); two saddle-node (SN, green and dark red); a SNIPER (green); and a homoclinic (HC, black dashed) bifurcation curve. The major difference, however, is that the PF bifurcation of the incoherent solution is supercritical for all parameter values $\Delta \geqslant 0, \omega_{0} \geqslant 0$. Moreover, the point A moves down in the parameter space away from the PF curve. There, it becomes a cusp point (CP), from which both $\mathrm{SN}$ curves (green and dark red) emerge. It is true that we still find a multistability region bounded by the SN and the HC curves, see also the inset in Fig. 7. Above the HB curve, there are two stable nontrivial fixed points, while below the HB curve a stable fixed point and a stable limit cycle coexist. However, we do not find stable solutions coexisting with the incoherent solution while being stable. Therefore, it is safe to argue that the symmetric trimodal network and the network of three coupled symmetric populations are not topologically equivalent.

\section{DISCUSSION AND CONCLUSION}

The Ott-Antonsen ansatz strongly boosted the analysis of Kuramoto models. Networks are assumed to consist of a continuum of oscillators, whose long-term dynamical behavior can be derived in the thermodynamic limit. Of particular interest for this paper is the extension to multiple coupled networks. A simple change of variables may transform two symmetrically coupled networks (with oscillators whose natural frequencies follow a unimodal distribution each) into one global network where the natural frequencies are drawn from a symmetric bimodal distribution. When assuming that internal and external coupling strengths in the two-population case differ, this transformation breaks down, and one is left with an additional degree of freedom. As we have proven in this paper, the additional parameter does not lead to new bifurcations but leaves both systems topologically equivalent. Stability, dynamics, and bifurcations of a symmetric twopopulation system of phase oscillators are equivalent to a single population with a bimodal frequency distribution. This topological equivalence can also be shown when introducing small symmetric time delays that allow for a phase-lag parameter reduction.

In the second part we aimed for generalizing the equivalence between multimodal and multiple coupled networks. However, already for the case of three subpopulations, where we adapted the same symmetry assumptions as in the twopopulation/bimodal case, this equivalence does no longer hold. Our symmetry assumptions are admittedly restrictive. Above all they only represent a slice of possible network configurations. That is, we cannot claim that the dynamics discussed here should be considered generic or not. However, our example clearly shows that the symmetric bidirectional coupling topology (cf. $K_{1,2}$ in Fig. 4) does not admit its dynamics to be described by a single network of oscillators whose natural frequencies follow a symmetric trimodal distribution. A detailed analysis in the presence of asymmetries in both the two-population/bimodal approach and the multiple populations/multimodal networks is beyond the scope of the present paper [31].

Throughout the paper we based our work on the original Kuramoto model, a network of phase oscillators that are all-to-all coupled through the sine of the pairwise phase differences. Coupling two of such networks leads to new long-term behavior such as partially synchronized states, so-called chimeras in the case of identical oscillators, see, 
e.g., Ref. [32]. Also, multistable regimes and oscillatory solutions are possible. For sure, nonlocal coupling, the introduction of phase-lag parameters as in Refs. [4,5,30], or more general time delays, see, e.g., Ref. [33], would have further enriched the dynamics. Recently, Martens, Bick, and Panaggio investigated how the introduction of heterogeneous phase lags in our two-population scenario of Sec. III shapes the dynamics. The additional control parameters were internal versus external phase-lag parameters next to (internal and external) coupling strengths and the intrinsic frequency $\omega$. Assuming only homogeneous oscillators in both populations renders the OA ansatz not applicable in a rigorous way. However, it has been argued that in the limit of zero width of the frequency distribution, $\Delta \rightarrow 0$, the assumption of nearly identical oscillators enabled the authors to analyze the system analytically [34]. Interestingly, they found chaotic attractors and resonance effects, which shows again the variety of dynamics of a mere two-population system, and highlights the importance to really understand their behavior.

In our two-population/bimodal scenarios the governing dynamics could be reduced to be effectively two dimensional. Hence, they cannot exhibit chaos. On the other hand, in the three-population/trimodal network chaotic trajectories should be possible. Though our focus is mainly on (disproving) the equivalence between the different approaches, a full picture should also take chaos in both systems into account by assessing maximal Lyapunov exponents [35]; see also Refs. [30,34].

Away from the symmetry assumptions considered throughout this work, but also when dealing with nonlocal coupling, phase-lag parameters, general time delay, or even finite-sized networks, i.e., in particular when the OA ansatz can no longer be applied, topological equivalences, or even (weaker) correspondences between multimodal and multiple coupled networks have to be demonstrated in order to show that coupled networks and networks with multimodal frequency distributions are equivalent. The analytic tractability of the Ott-Antonsen ansatz helped us to rigorously prove first results about similarities and differences between these two approaches. We believe that, despite the limited range of application of such models, our findings can be assumed seminal for a broader variety of models, and therefore will further enlighten the view on an accurate interchangeability of multimodal networks and coupled unimodal networks, which in the end will increase the flexibility to derive and specify models in diverse fields of applications.

\section{ACKNOWLEDGMENTS}

We thank Bob van Dijk for interesting discussions, and the anonymous reviewers for their helpful comments and for pointing us to Refs. $[6,27,33]$. This project has received funding form the European Union's Horizon 2020 research and innovation program under the Marie Skłodowska-Curie Grant Agreement No. 642563 (COSMOS).

\section{APPENDIX A: BIFURCATION SCHEME FOR TWO COUPLED NETWORKS}

We give a comprehensive overview of the bifurcation scheme of system (9). Recall that $\Delta, \omega_{0}$, and $\kappa$ are the (scaled) parameters denoting the distribution widths, the

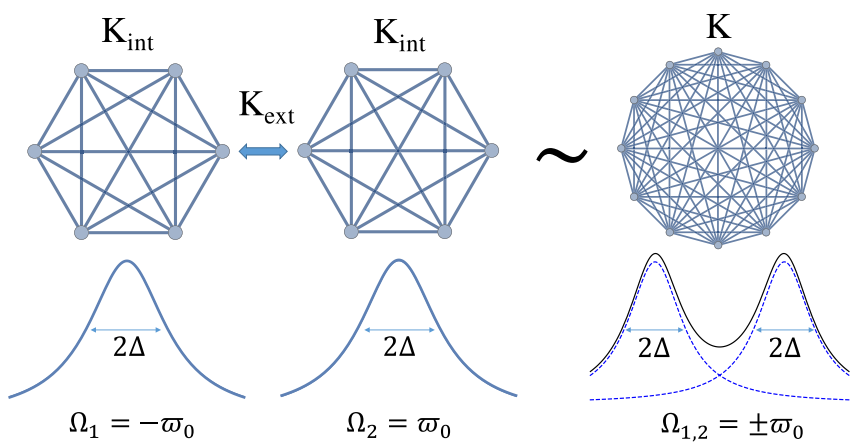

FIG. 8. Two all-to-all coupled networks (left) with unimodal frequency distributions each; a single all-to-all coupled network (right) with a symmetric bimodal frequency distribution function; cf. main text Eqs. (5) and (6) where details are provided.

distance between the peaks of the distribution functions, and the ratio of external to internal coupling, respectively. Since $q=\rho^{2}$ denotes the squared (local) order parameter of each subpopulation, for the solutions of interest we have $0 \leqslant q \leqslant 1$ and all the parameters being non-negative. We also note that for $\kappa=0$, the external coupling $K_{\text {ext }}$ vanishes, therefore leaving two separate, noninteracting networks; see Fig. 8.

In line with the main text, the red plane in Fig. 9 displays the supercritical Hopf bifurcation while the orange cone represents the transcritical bifurcation. Between the green curves (exact formulas in the main text) we find the saddle-node plane, which denotes the parameter values, for which a pair of a stable fixed point and a saddle point emerges as a neutral fixed point.

Along the saddle-node plane, however, we have to distinguish two cases of this bifurcation. For all points on the plane with $\Delta$ bigger than some critical value $\Delta_{c}$, the neutral fixed point emerges away from the stable limit cycle (for $\Delta \leqslant 1)$, or away from the stable incoherent solution $(\Delta \geqslant 1)$. For $\Delta \leqslant \Delta_{c}<1$ the creation of that fixed point takes place directly on the limit cycle, where $\Delta_{c}$ denotes the value for the codimension 2 bifurcation points (blue) on the green plane in Fig. 9-for $\kappa=1$ this critical parameter is $\Delta=\Delta_{c} \approx 0.7384$. In particular, the emergent fixed point is about to split into a pair of a stable fixed point and a saddle point, therefore it

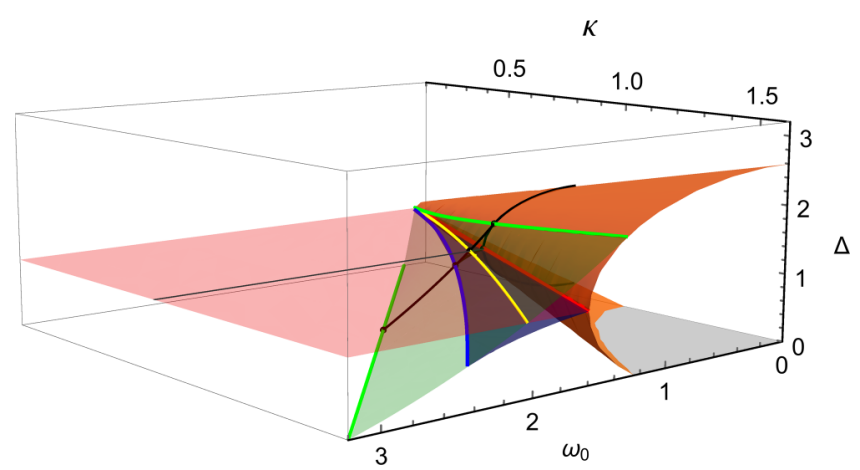

FIG. 9. Bifurcation boundaries (back view of Fig. 1). Red plane: Hopf; orange cone: transcritical; green plane (within green lines): saddle node; blue: homoclinic bifurcation. Blue line: Saddle-node loop curve; yellow: intersection of Hopf and SN; black lines: cross section at $\kappa=0.8$, see also Fig. 10 . 

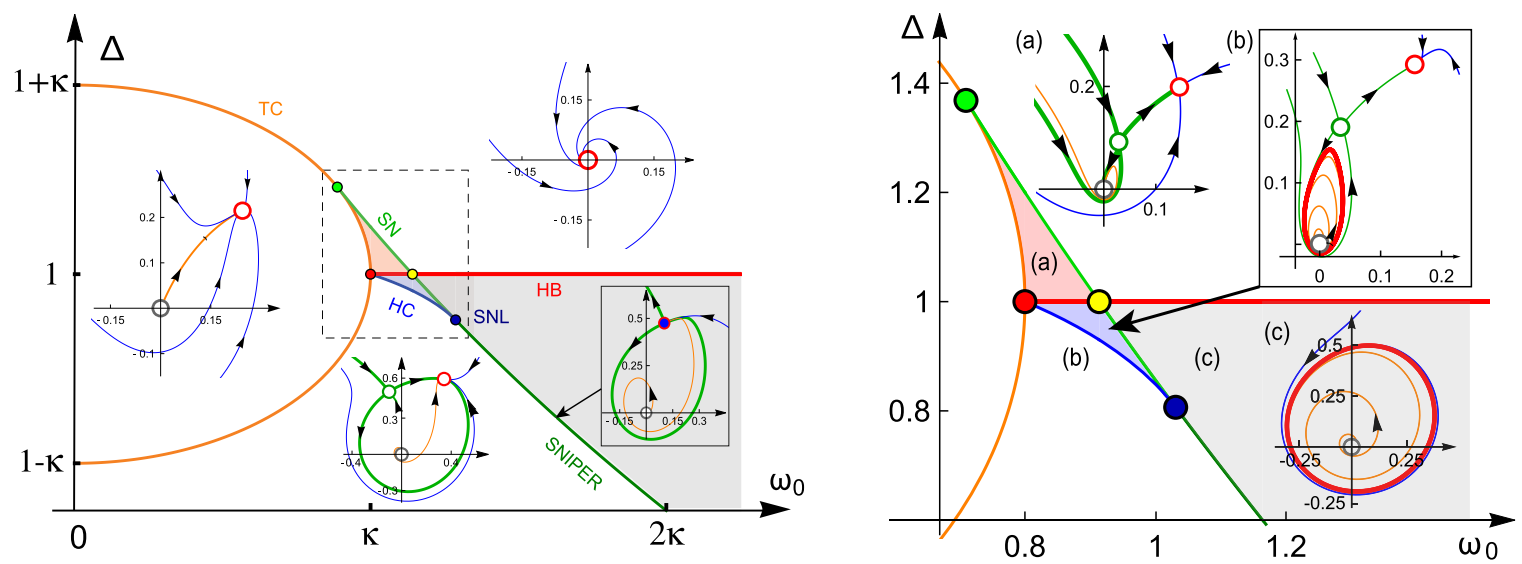

FIG. 10. Bifurcation boundaries (left) and bistability region (dashed, right): cross section of Fig. 9 at $\kappa<1$. Red: Hopf; orange: transcritical; green: saddle node; blue: homoclinic; blue point: saddle-node loop bifurcation. Insets: $(q, \psi)$-phase portraits (in polar coordinates) in their specific parameter regions, red circle: stable; gray: unstable fixed point; green: saddle point. The right figure is a detailed view of the dashed box in the left figure.

destroys the limit cycle by forcing the period to infinity. This is a saddle-node infinite-period bifurcation (SNIPER). The (blue) critical curve $\Delta_{c}=\Delta_{c}\left(\omega_{0}, \kappa\right)$, which separates the two types of saddle-node bifurcations, consists of saddle-node loop bifurcation points, i.e., bifurcation points of codimension 2 .

Furthermore, numerics reveals a plane connecting the (blue) saddle-node loop curve with the (red) curve $\left\{\Delta=1, \kappa=\omega_{0} \mid \kappa, \omega_{0} \geqslant 0\right\}$. The latter curve comprises the parameter values for which the saddle point (emerging from the saddle-node bifurcation) collapses with the stable incoherent solution, which then becomes unstable. Along the blue plane in Fig. 9, a homoclinic bifurcation takes place. Here, the saddle point approaches the limit cycle, which is therefore destroyed in the end. Figure 10 displays the cross section at $\kappa=0.8$ of the three-dimensional bifurcation boundaries, and elucidates the generic dynamical behavior within the corresponding parameter regions. Note that this cross section is representative for all $\kappa>0$ as has been proven in the main text. Unfortunately, analytical formulas for the homoclinic and saddle-node loop bifurcations are still missing both in the bimodal case as well as in the subpopulation approach, so that we here rely on the numerics.

\section{APPENDIX B: INFLUENCE OF TIME DELAY ON THE EFFECTIVE COUPLING STRENGTHS}

An alternative case for deriving the main text's Eq. (9) is to introduce delays. For this we consider two subpopulations of oscillators that follow a unimodal frequency distribution with distinct peaks as in the left part of Fig. 8. We assume again all-to-all coupling, yet this time with the same coupling strength $K=K_{\text {int }}=K_{\text {ext }}$ within as well as across subpopulation boundaries. By contrast, however, we introduce population-specific time delays $\tau_{\sigma, \sigma^{\prime}}$, such that the dynamics of the $k$ th oscillator of population $\sigma$ is governed by

$$
\dot{\theta}_{\sigma, k}(t)=\omega_{\sigma, k}+\frac{K}{N} \sum_{\sigma^{\prime}=1}^{2} \sum_{j=1}^{N} \sin \left[\theta_{\sigma^{\prime}, j}\left(t-\tau_{\sigma^{\prime}, \sigma}\right)-\theta_{\sigma, k}(t)\right]
$$

where $k=1, \ldots, N$ and $\sigma=1,2$. For simplicity, we consider the time delay within populations to be negligible compared to that across boundaries, hence without loss of generality we have $\tau_{\sigma, \sigma^{\prime}}=0$ and $\tau_{\sigma, \sigma}=\tau>0$. If we further assume the time delay $\tau \ll 2 \pi / \omega_{0}$ and also the coupling strength $K$ being sufficiently small, we can approximate $\theta_{\sigma^{\prime}, j}(t-\tau)$ by means of Taylor such that

$$
\begin{aligned}
\theta_{\sigma^{\prime}, j}(t-\tau) & \approx \theta_{\sigma^{\prime}, j}(t)-\tau \dot{\theta}_{\sigma^{\prime}, j}(t) \\
& \approx \theta_{\sigma^{\prime}, j}(t)-\tau\left[\bar{\omega}_{\sigma^{\prime}}+O(K)\right] \\
& \approx \theta_{\sigma^{\prime}, j}(t)-\alpha_{\sigma^{\prime}}
\end{aligned}
$$

where $\alpha_{\sigma^{\prime}}=\tau \bar{\omega}_{\sigma^{\prime}}$ with $\bar{\omega}_{\sigma^{\prime}}= \pm \varpi_{0}$. Hence, we can replace the time delay by phase lag parameters, cf. [36,37]. The population-specific phase-lag parameters depend on the mean frequency of the oscillators, such that we have $\gamma:=\alpha_{1}=-\alpha_{2}$. Consequently, the governing equations read

$$
\begin{array}{r}
\dot{\theta}_{1, k}=\omega_{1, k}+\frac{K}{N} \sum_{j=1}^{N} \sin \left(\theta_{1, j}-\theta_{1, k}\right) \\
+\frac{K}{N} \sum_{j=1}^{N} \sin \left(\theta_{2, j}-\theta_{1, k}-\gamma\right) \\
\dot{\theta}_{2, k}=\omega_{2, k}+\frac{K}{N} \sum_{j=1}^{N} \sin \left(\theta_{2, j}-\theta_{2, k}\right) \\
+\frac{K}{N} \sum_{j=1}^{N} \sin \left(\theta_{1, j}-\theta_{2, k}+\gamma\right) .
\end{array}
$$

Now, we use again the Ott-Antonsen ansatz and perform the same steps for this system as we have done in the main text. We end up with the dynamics for the local order parameters

$$
\begin{aligned}
\dot{z}_{\sigma}= & -\left(\Lambda_{\sigma}-i \Omega_{\sigma}\right) z_{\sigma}+\frac{K}{2} z_{\sigma}\left(1-\left|z_{\sigma}\right|^{2}\right) \\
& +\frac{K}{2} e^{-i \gamma}\left(z_{\sigma^{\prime}}-z_{\sigma}^{2} z_{\sigma^{\prime}}^{*} e^{2 i \gamma}\right)
\end{aligned}
$$


where we have let the oscillators' frequencies follow symmetric Lorentzians, $g_{\sigma}(\omega)=\left(\Lambda_{\sigma} / \pi\right) /\left\{\left[\left(\omega-\Omega_{\sigma}\right)^{2}+\Lambda_{\sigma}^{2}\right]^{2}\right\}$, with $\Lambda_{1}=\Lambda_{2}=: \Lambda$ and $\Omega_{1}=\varpi_{0}=-\Omega_{2}$. As in the main text, we further introduce polar coordinates for the local order parameters, $z_{\sigma}=\rho_{\sigma} e^{i \phi_{\sigma}}$. For symmetry reasons we set $\rho:=$ $\rho_{1}=\rho_{2}$, and let $\Psi$ denote the difference of the mean phases. Then, we scale according to $\tilde{t}=\frac{K}{2} t, \Delta=\frac{4 \Lambda}{K}, \omega_{0}=\frac{4 \varpi_{0}}{K}$ and write $q=\rho^{2}$. Hence, we arrive at

$$
\begin{aligned}
\dot{q} & =q[1-\Delta-q+(1-q) \cos (\psi+\gamma)] \\
\dot{\psi} & =\omega_{0}-(1+q) \sin (\psi+\gamma),
\end{aligned}
$$

where the dot notation refers to the derivative with respect to $\tilde{t}$. In particular, this is exactly the same system (9) as in the main text for $\kappa=1$, and as Eqs. (25) and (26) in Martens et al. [9]. Merely, the mean phase difference is shifted by the (constant) phase-lag parameter $\gamma$. But due to the $2 \pi$ periodicity of $\Psi$, this phase shift does not alter the original dynamics.

Therefore, we may conclude that population-specific time delay does not change the effective coupling strengths, as would have led to $\kappa \neq 1$ in system (9). Note, however, that our derivation was based on a restricted size of time delays. If allowing more general time delays, the phase lag approximation cannot hold anymore. As has been shown, e.g., by Yeung and Strogatz in Ref. [38], already one single network with time delay can exhibit bistability and an oscillating order parameter. Hence, we may expect that the dynamics of two coupled networks may reveal even more delicate synchronization patterns, which await to be explored.
[1] J. A. Acebrón, L. L. Bonilla, C. J. Pérez Vicente, F. Ritort, and R. Spigler, Rev. Mod. Phys. 77, 137 (2005).

[2] F. A. Rodrigues, T. K. D. Peron, P. Ji, and J. Kurths, Phys. Rep. 610, 1 (2016).

[3] E. Ott and T. M. Antonsen, Chaos 18, 037113 (2008).

[4] D. M. Abrams, R. Mirollo, S. H. Strogatz, and D. A. Wiley, Phys. Rev. Lett. 101, 084103 (2008).

[5] E. Montbrió, J. Kurths, and B. Blasius, Phys. Rev. E 70, 056125 (2004).

[6] E. Barreto, B. Hunt, E. Ott, and P. So, Phys. Rev. E 77, 036107 (2008); P. So, B. C. Cotton, and E. Barreto, Chaos 18, 037114 (2008).

[7] C. R. Laing, Chaos 19, 013113 (2009).

[8] Y. Kawamura, H. Nakao, K. Arai, H. Kori, and Y. Kuramoto, Chaos 20, 043110 (2010).

[9] E. A. Martens, E. Barreto, S. H. Strogatz, E. Ott, P. So, and T. M. Antonsen, Phys. Rev. E 79, 026204 (2009).

[10] Y. Kuramoto, Chemical Oscillations, Waves, and Turbulence (SIAM, Philadelphia, 1984).

[11] S. H. Strogatz, Physica D 143, 1 (2000).

[12] K. Okuda and Y. Kuramoto, Prog. Theor. Phys. 86, 1159 (1991).

[13] J. D. Crawford, J. Stat. Phys. 74, 1047 (1994).

[14] D. M. Abrams and S. H. Strogatz, Phys. Rev. Lett. 93, 174102 (2004).

[15] H. Sakaguchi, Prog. Theor. Phys. 79, 39 (1988).

[16] J. Keizer, Statistical Thermodynamics of Nonequilibrium Processes (Springer, Berlin, 1987).

[17] S. H. Strogatz and R. E. Mirollo, J. Stat. Phys. 63, 613 (1991).

[18] E. Ott and T. M. Antonsen, Chaos 19, 023117 (2009).

[19] B. Sonnenschein and L. Schimansky-Geier, Phys. Rev. E 88, 052111 (2013).

[20] E. Ott, B. R. Hunt, and T. M. Antonsen, Chaos 21, 025112 (2011).

[21] B. Pietras and A. Daffertshofer, Chaos 26, 103101 (2016).
[22] We consider $K_{\text {int }} \neq 0$ and note that the scaling does not affect the quality of bifurcations, i.e., the original and scaled systems are topologically equivalent.

[23] Our unscaled system (8) is an exact representation of Eqs. (22) and (23) for $\tilde{K}=K / 2$ in the notation used in Ref. [9].

[24] The absolute value of the global order parameter $z$ reads in general: $\quad R=|z|=\frac{1}{2}\left|\rho_{1} \mathrm{e}^{i \phi_{1}}+\rho_{2} \mathrm{e}^{i \phi_{2}}\right|=$ $\frac{1}{2} \sqrt{\rho_{1}^{2}+\rho_{2}^{2}+2 \rho_{1} \rho_{2} \cos \left(\phi_{2}-\phi_{1}\right)}$.

[25] D. Anderson, A. Tenzer, G. Barlev, M. Girvan, T. M. Antonsen, and E. Ott, Chaos 22, 013102 (2012).

[26] M. Komarov and A. Pikovsky, Phys. Rev. E 84, 016210 (2011).

[27] J. A. Acebrón, A. Perales, and R. Spigler, Phys. Rev. E 64, 016218 (2001).

[28] $\varpi_{0}$ is assumed to be sufficiently large to guarantee isolated peaks and all distributions have width $\Lambda$.

[29] The symmetric trimodal distribution features three peaks of the distribution function that have the same height. Our notion is not to be mistaken with the case where three symmetric unimodal distributions, i.e., identical widths and centers symmetrically arranged, sum equally up to form the trimodal distribution. Then, the middle peak is dominant, which we prevent by weighting the central distribution less than the outer two.

[30] E. A. Martens, Phys. Rev. E 82, 016216 (2010).

[31] B. Pietras, N. Deschle, and A. Daffertshofer (unpublished).

[32] D. Pazó and E. Montbrió, Phys. Rev. X 4, 011009 (2014).

[33] W. S. Lee, E. Ott, and T. M. Antonsen, Phys. Rev. Lett. 103, 044101 (2009).

[34] E. A. Martens, C. Bick, and M. J. Panaggio, Chaos 26, 094819 (2016).

[35] A. Pikovsky and A. Politi, Lyapunov Exponents: A Tool to Explore Complex Dynamics (Cambridge University Press, Cambridge, 2016).

[36] E. M. Izhikevich, Phys. Rev. E 58, 905 (1998).

[37] M. J. Panaggio and D. M. Abrams, Nonlinearity 28, R67 (2015).

[38] M. K. Stephen Yeung and S. H. Strogatz, Phys. Rev. Lett. 82, 648 (1999). 